\author{
EVS27 \\ Barcelona, Spain, November 17-20, 2013
}

\title{
Analysis and Simulation of a novel HEV using a Single Electric Machine
}

\author{
Futang Zhu, Chengliang Yin, Li Chen, Cunlei Wang \\ National Engineering Laboratory for Automotive Electronic Control Technology, Shanghai Jiao Tong University, \\ Dongchuan Road 800, Shanghai, 200240, P.R. China. Email: zhu futang@hotmail.com
}

\begin{abstract}
This paper presents the design and validation of a novel multi-mode transmission (MMT) for a hybrid electric vehicle (HEV) using a single electric machine (EM), which implies compact and low cost. The topology of the MMT planetary gearset is the same as that of conventional four speed automatic transmissions. The MMT uses two planetary gearsets and four clutches to combine an engine and an EM with the vehicle, and the EM is operated as a motor or a generator. The MMT realizes five power flow modes, which are developed into sixteen operating modes, including one motor-only mode, four engineonly modes, four compound driving modes, six braking modes, and one charging while parking mode. The single EM HEV solution avoids losses from another EM and extra power electronics which are employed in many existing HEVs. The properly arranged clutches transmit power flow more flexibly, allow direct mechanical power transmission from the engine to the drive shaft, and avoid spin loss for the engine and energy conversion loss for the electric components. The fuel consumption of the proposed hybrid vehicle system is compared with a benchmark vehicle which uses a planetary gearset, two EMs and no clutch. Simulation results under New European Driving Cycle show that the fuel consumption of the proposed HEV is comparable to the benchmark vehicle. Reasons for comparable results are explored, which indicates the fuel economy potential of the new concept.
\end{abstract}

Keywords: multi-mode transmission, single electric machine, hybrid electric vehicle, planetary gearset

\section{Introduction}

Hybrid electric vehicles (HEVs), as one kind of new generation vehicles, are considered to offer the best promise in the short to mid-term [1] to solve the problem of global energy shortage and environment pollution. For most of the currently developed HEVs, such as TOYOTA THS, Ford FHS, GM AHS, etc, two electric machines (EMs) are commonly used [2-5]. One of the two EMs works mostly as a driving motor, the other as a generator. However, an EM can be operated in any one of the four quadrants of the torque versus speed coordinate system. The EM works as a motor in the quadrant 1 and 3, and as a generator in 2 and 4. Some researchers are enlightened to investigate the potential that a single EM helps the $\mathrm{HEV}$ realize necessary operating modes and gain comparable performance to double EM HEVs.

The single EM HEV uses one less EM and its associated power electronics system, which implies advantages of compact, low power loss and low cost. Several single EM HEV schemes 
were investigated and many of them exhibit comparable performance to double EM schemes [6-9]. However, the single EM cannot work as a motor and a generator at the same time, thus cannot modulate the engine speed and torque simultaneously like in a double EM scheme, which introduces limitation to the HEV performance. In most cases, this comprise in HEV performance can be counterbalanced by the power loss reduction in single EM scheme. While under some driving conditions, for example in high velocities, the overall powertrain efficiency would deteriorate largely in single EM scheme, which won't necessarily be compensated by its power loss reduction. Therefore, the fuel consumption comparison and analysis should be carried out in synthetic driving cycles to investig ate its application potential.

This paper presents a novel multi-mode transmission (MMT) for the single EM HEV. It contains two planetary gearsets and four clutches. The topology of the MMT is the same as that of the popular four speed ATs, such as GM (4T65E, 4L60E), CHR YSLER (42LE), ZF (4HP-18) and so on [10]. We believe the proposed architecture is more viable than other transmissions that are newly designed for practical applications because of its evolutionary nature.

In this paper, a mathematical model developed in MATLAB/Simulink for the proposed HEV is used to simulate and analyze its performance. Section 2 explains the system architecture and operating modes, following the energy management strategy, including mode selection, and torque distribution. Finally, the application potential is discussed and concluded based on the simulation results comparison with those of a second generation THS-II HEV under the New European Driving Cycle (NEDC).

\section{System Architecture Operating Modes}

\subsection{System Architecture}

The MMT consists of two planetary gearsets, called input and output respectively. The carrier gear $\mathrm{C} 1$ of input planetary gearset is connected to the ring gear R2 of output planetary gearset, and the ring gear R1 of input planetary gearset is connected to the carrier gear $\mathrm{C} 2$ of output planetary gearset. Four clutches are employed, two of them are rotating clutches (denoted as CR1, CR2, respectively), and the other two are braking clutches (denoted as CB1, CB2, respectively).

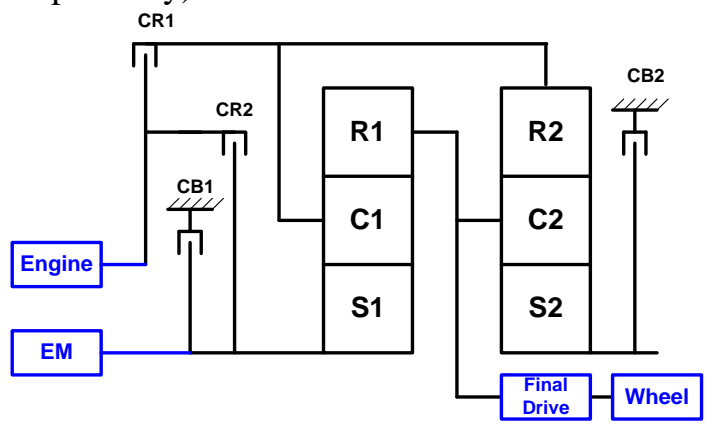

Figure1: Schematic diagram of the proposed single EM HEV

The single EM HEV has two power sources, the engine and the EM, as illustrated in Fig.1. The engine is connected to the two planetary gearsets through CR1 and CR2. The EM is connected to the sun gear S1 directly, and the motion of the EM or $\mathrm{S} 1$ is manipulated by CB1 and CR2.

\subsection{Ope rating Modes}

The proposed MMT can realize five basic power flow modes, i.e., Motor_only driving, Engine_only driving, Compound driving, Braking, and Charging while parking. The power flow modes could be realized by different transmission ratios which are changed through clutch engagement and disengagement.

Table1: Operating modes of the single EM HEV

\begin{tabular}{|c|c|c|c|c|c|c|}
\hline \multirow{2}{*}{$\begin{array}{c}\text { Power } \\
\text { flow } \\
\text { mode }\end{array}$} & \multirow{2}{*}{$\begin{array}{l}\text { Operating } \\
\text { Mode }\end{array}$} & \multicolumn{4}{|c|}{ Clutch Operation } & \multirow{2}{*}{$\begin{array}{c}\text { EM } \\
\text { Operati } \\
\text { on }\end{array}$} \\
\hline & & $\begin{array}{c}\mathrm{CB} \\
1\end{array}$ & $\begin{array}{c}\text { CB } \\
2\end{array}$ & $\begin{array}{c}\mathrm{CR} \\
1\end{array}$ & $\begin{array}{c}\mathrm{CR} \\
2\end{array}$ & \\
\hline $\begin{array}{l}\text { Motor_on } \\
\text { ly driving }\end{array}$ & Motor_only & & $\diamond$ & & & mot \\
\hline $\begin{array}{c}\text { Engine_o } \\
\text { nly } \\
\text { driving }\end{array}$ & $\begin{array}{l}\text { Engine_only_1 } \\
\text { Engine_only_2 } \\
\text { Engine_only_3 } \\
\text { Engine_only_4 }\end{array}$ & $\bullet$ & 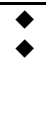 & $\bullet$ & $\bullet$ & $\begin{array}{c}\text { spinning } \\
\text { spinning } \\
\text { spinning } \\
\text { fixed }\end{array}$ \\
\hline $\begin{array}{l}\text { Compoun } \\
\text { d driving }\end{array}$ & $\begin{array}{c}\text { Compound_1 } \\
\text { Compound_2 } \\
\text { Compound_3 } \\
\text { Compound_4_EV } \\
\text { T }\end{array}$ & & 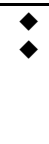 & $\bullet$ & $\bullet$ & $\begin{array}{l}\text { mot/gen } \\
\text { mot/gen } \\
\text { mot/gen } \\
\text { mot/gen }\end{array}$ \\
\hline Braking & $\begin{array}{c}\text { Mech_Braking } \\
\text { Reg_Braking } \\
\text { Compound_Braki } \\
\text { ng_0 } \\
\text { Compound_Braki } \\
\text { ng_1 } \\
\text { Compound_Braki } \\
\text { ng_2 } \\
\text { Compound_Braki } \\
\text { ng_3 }\end{array}$ & & $\bullet$ & $\diamond$ & $\bullet$ & $\begin{array}{c}\text { fixed } \\
\text { gen } \\
\text { gen } \\
\text { gen } \\
\text { gen } \\
\text { gen }\end{array}$ \\
\hline $\begin{array}{l}\text { Charging } \\
\text { while } \\
\text { parking }\end{array}$ & $\begin{array}{c}\text { Charging while } \\
\text { parking }\end{array}$ & & & & $\diamond$ & gen \\
\hline
\end{tabular}

[Note]mot:motor; gen: generator

Considering various combination of clutch operations, the power flow modes are further developed into sixteen operating modes, as shown 
in Table 1, where ' denotes the locked phase of a clutch.

A control-oriented MMT model capturing the driveline dynamics is developed, which can be referred to [11].

\section{Energy Management Strategy}

The proposed energy management system consists of two modules: mode selection and torque distribution, as shown in Fig. 2. The mode selection module selects a proper mode from the feasible modes, which are defined by the number of clutch operations. The torque distribution module distributes the optimal torque commands to the engine and EM.

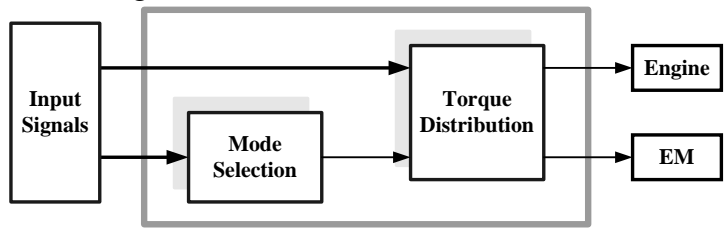

Figure2: Schematic diagram of the proposed single EM HEV

\subsection{Mode Selection}

The inputs of the mode selection module are the required torque, vehicle states and battery state of charge (SOC). Extensive algorithms have been applied in estimation and control development, such as fuzzy logic [12], optimization methods [13] and neural networks [14]. Still, rule-based control strategies, which are developed based on engineering intuition and simple analysis of component-efficiency tables, are widely used in many HEVs due to its ease in handing changes in the operating mode.

In this paper, a rule-based control strategy is developed for mode selection. Engine is the primary power source and EM is the supplemental power source. Still, the engine and the EM can drive the vehicle independently. The control strategy aims to operate the engine working in the high efficiency or sub-high efficiency range, through the following control rules. The rules are implemented in MATLAB Stateflow, and an example for propulsion case is given in Fig. 3. The detailed control rules are presented below:

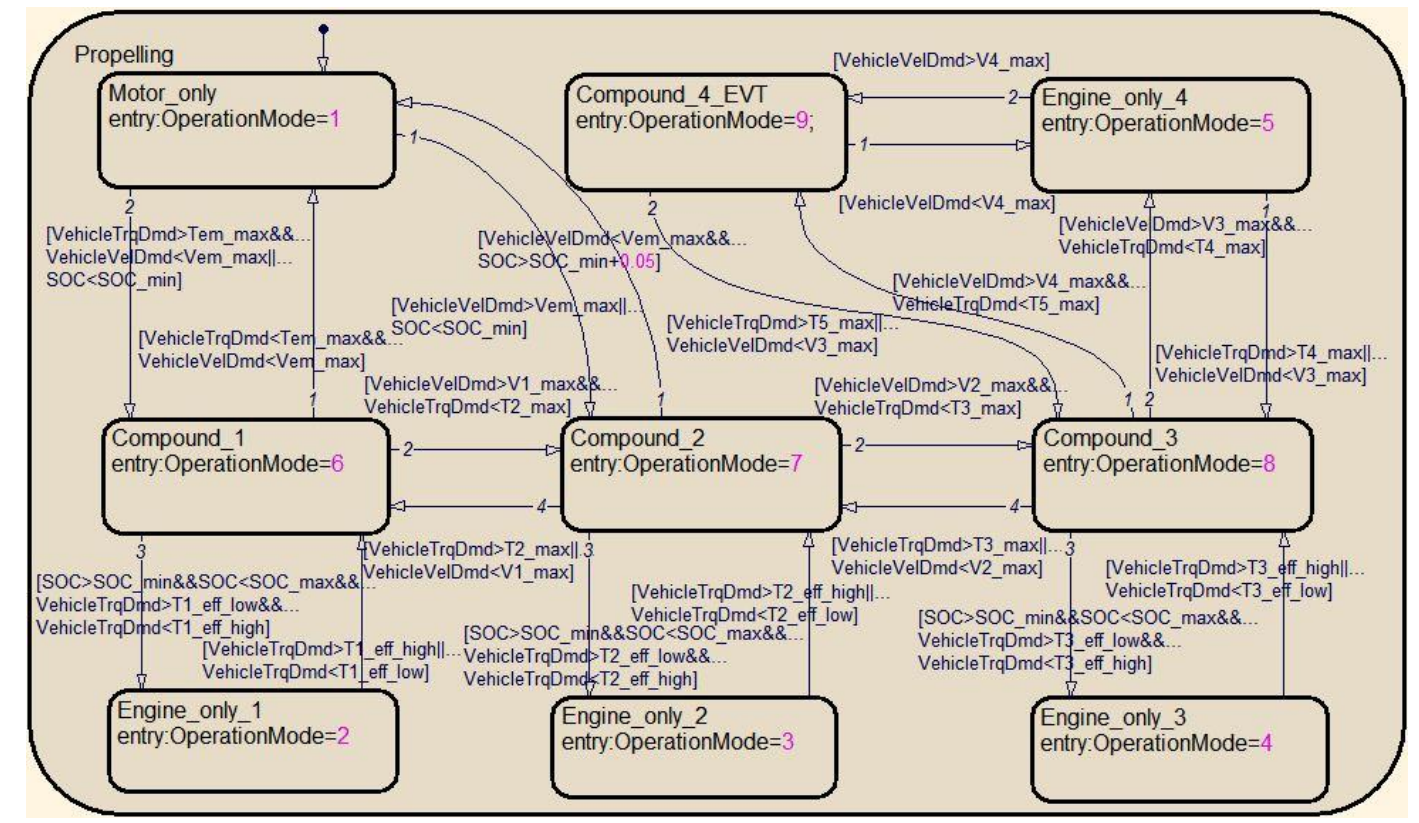

Figure3: Stateflow chart of the energy management controller

(1) To avoid inefficient engine operations, the engine shuts off and the electric motor supplies all driving torque (i.e., use Motor_only mode) below a certain vehicle velocity denoted by $v_{\_}$em_max. The required driving torque is within the EM torque capacity, calculated from $T_{\mathrm{em} \_ \text {max }}$ and the gear ratio.

(2) When the required torque exceeds the EM capacity, the engine is started up using Compound_1 mode when the desired velocity is below $v_{-}$em_max, or using Compound_2 mode when the velocity is above $v_{-}$em_max or the SOC is below the lower limit $S O C_{\text {min. }}$. And then, EM provides assistive torque to make the engine operate in high or sub-high efficiency area. The selection among the four compound driving modes basically depends on the vehicle velocity and the required driving torque. Compound_1 mode is selected when the vehicle velocity is below v1_max which is calculated from $\omega_{\text {up }}$ and the first gear ratio, and the 
required driving torque is above $T_{2}$ max, which is calculated from $T_{\text {em_max }}, T_{\text {e_up }}$ and the second gear ratio. Similar rules are designed for selecting Compound_2, Compound_3 and Compound_4_EVT mode.

(3) When the battery SOC is below the maximum limit and the required torque does not exceed the maximum torque of the high efficiency region, the engine provides additional torque which passes through EM to charge the battery.

(4) When the battery SOC is within the $S O C_{\min }$ and $S O C_{\max }$, and the engine can work in high or sub-high efficiency region [ $T$ eng_only_low, $T$ eng_only_up], EM does not work and the HEV is operated in one of the four Engine_only driving modes. The selection of the four modes basically depends on the vehicle velocity and the required torque, which is the same as that for a traditional engine driven vehic le.

(5) When the required torque is negative and SOC is below SOC $\max$, Reg_braking mode is activated. Since the regenerative braking power is limited by the rated capacity of the battery, the regenerative braking torque denoted by $T_{\text {reg_lim }}$ is also limited, and calculated from $T_{\text {em_max }}$ and the gear ratio.

(6) When the required braking torque is more negative than $T_{\text {reg_lim }}$ and $S O C$ is below $S O C_{\max }$, the mechanical braking system provides the additional braking torque in Compound_braking_0 mode.

(7) When the required braking torque is more negative than the summation of $T_{\text {re__lim }}$ and the mechanical braking system, the engine enhances braking by its friction and air resistance, in Compound_braking_1, 2 or 3 modes. Since Compound_braking_1 mode has a bigger amplifier ratio, the braking torque generated by the engine resistance is larger than the other two modes. When $\mathrm{SOC}$ reaches $S O C_{\max }$, regenerative braking is not used for battery durability consideration, i.e., $T_{\text {reg_lim }}$ is zero. Thus, the braking levers on the mechanical system and the engine.

(8) In order to maintain battery capacity, the engine is allowed to charge the battery when the vehicle is parking. In this way, the battery can be always ready whenever the vehicle begins to run (Charging while parking mode)

\subsection{Torque Distribution}

The relationship between the MMT input torques and output is specified using the following dynamical equation.

$$
\left(\begin{array}{l}
T_{1} \\
T_{2} \\
T_{3}
\end{array}\right)=\left(\begin{array}{lll}
I_{11} & I_{12} & I_{13} \\
I_{21} & I_{22} & I_{23} \\
I_{31} & I_{32} & I_{33}
\end{array}\right) \times\left(\begin{array}{c}
\dot{\omega}_{\text {eng }} \\
\dot{\omega}_{\text {em }} \\
\dot{\omega}_{\text {out }}
\end{array}\right)
$$

or

$$
\left(\begin{array}{l}
\dot{\omega}_{\text {eng }} \\
\dot{\omega}_{\text {em }} \\
\dot{\omega}_{\text {out }}
\end{array}\right)=\frac{1}{J_{\text {det }}}\left(\begin{array}{ccc}
J_{11} & J_{12} & J_{13} \\
J_{21} & J_{22} & J_{23} \\
J_{31} & J_{32} & J_{33}
\end{array}\right) \times\left(\begin{array}{c}
T_{1} \\
T_{2} \\
T_{3}
\end{array}\right)
$$

where

$$
\begin{gathered}
T_{1}=T_{\text {eng }}-T_{C R 1}-T_{C R 2} \\
T_{2}=T_{\text {em }}-T_{\text {out }}+T_{C R 1}+T_{C R 2}+T_{C B 1}+T_{C B 2} \\
T_{3}=\left(1+R_{a}\right) T_{e m}+T_{C R 1}+\left(1+R_{a}\right)\left(T_{C R 2}+T_{C B 1}\right)-R_{b} T_{C B 2} \\
I_{11}=I_{\text {eng }} ; I_{12}=I_{13}=I_{21}=I_{31}=0 ; I_{22}=I_{S 1}+I_{C 1} \cdot 1 /\left(1+R_{a}\right) \\
-I_{S 2} \cdot R_{b} /\left(1+R_{a}\right)+I_{R 2} \cdot 1 /\left(1+R_{a}\right) ; I_{23}=I_{C 1} \cdot R_{a} /\left(1+R_{a}\right)+ \\
I_{R 1}+I_{S 2} \cdot\left(1+R_{a}+R_{b}\right) /\left(1+R_{a}\right)+I_{C 2}+I_{R 2} \cdot R_{a} /\left(1+R_{a}\right) ; \\
I_{32}=I_{S 1} \cdot\left(1+R_{a}\right)+I_{C 1} \cdot 1 /\left(1+R_{a}\right)+I_{S 2} \cdot R_{a} R_{b} /\left(1+R_{a}\right) \\
+I_{R 2} \cdot 1 /\left(1+R_{a}\right) ; I_{33}=I_{C 1} \cdot R_{a} /\left(1+R_{a}\right)- \\
I_{S 2} \cdot\left(1+R_{a}+R_{b}\right) R_{b} /\left(1+R_{a}\right)+I_{R 2} \cdot R_{a} /\left(1+R_{a}\right) \\
\text { and } \\
J_{11}=I_{22} \times I_{33}-I_{23} \times I_{32} ; J_{12}=-\left(I_{21} \times I_{33}-I_{23} \times I_{31}\right) ; \\
J_{13}=I_{21} \times I_{32}-I_{22} \times I_{31} ; J_{21}=-\left(I_{12} \times I_{33}-I_{13} \times I_{32}\right) ; \\
J_{22}=I_{11} \times I_{33}-I_{13} \times I_{31} ; J_{23}=-\left(I_{11} \times I_{32}-I_{12} \times I_{31}\right) ; \\
J_{31}=I_{12} \times I_{23}-I_{13} \times I_{22} ; J_{32}=-\left(I_{11} \times I_{23}-I_{13} \times I_{21}\right) ; \\
J_{33}=I_{11} \times I_{22}-I_{12} \times I_{21} ; \\
J_{\text {det }}=I_{11} \times J_{11}+I_{12} \times J_{12}+I_{13} \times J_{13}
\end{gathered}
$$

The torque distribution module solves this equation to get the input torques based on measured data and predefined parameters. The left side of (1) involves seven torques, i.e., $T_{e n g}, T_{e m}$, Tout and four clutch torques. Tout is the actual torque with respect to the $\mathrm{C} 2$ shaft, and is supposed to be equal to Toutreq, which comes from a predefined required torque. $T_{e n g}$ and $T_{e m}$ are to be decided by this torque distribution module. The modes with the same clutch status have the same expression for $T_{e n g}$ and $T_{e m}$ calculated from (1). So the solutions for the 16 operation modes are classified in terms of the clutch status. The clutches are either locked or open when the MMT is in one mode. The transmitted torque is zero when the clutch is open, and is an internal torque when locked. Therefore, the expressions of $T_{e n g}$ and $T_{e m}$ in each mode can be derived applying the above principals.

\section{Simulation and Analysis}

The performance of the proposed MMT vehicle is demonstrated by comparing against a "THS IIlike" vehicle which uses two electric machines without a clutch but else all the vehicle parameters are the same as the target vehicle studied in this paper. The diagram of the THS II electric variable 
transmission is shown in Fig. 4. The second planetary gearset is considered as a reduction gear because its carrier is fixed. The way to connect the engine, EM1 of the "THS II-like" vehicle is the same as that of the proposed HEV. The New European Driving Cycle (NEDC) comprehensive driving cycle which consists of four identical urban cycles and one extra-urban cycle with frequent start/stop is used to assess the vehicle performance.

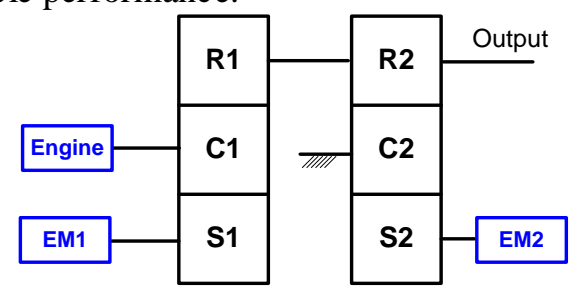

Figure4: Schematic diagram of the benchmark "THS II-like" vehicle

To reduce fuel consumption, the engine used in the MMT vehicle is downsized from $80 \mathrm{~kW}$ to $57 \mathrm{~kW}$. The engine is augmented by EM, which has a maximum power of $30 \mathrm{~kW}$. A Lithium Iron Phosphate battery is used as the energy storage system. The maximum power of EM1 and EM2 in the "THS II-like" vehic le is $15 \mathrm{~kW}$ and $30 \mathrm{~kW}$, respectively.

\subsection{Simulation Results}

The engine, battery and vehicle parameters of the proposed MMT vehicle and the "THS II-like" vehicle are the same. The two vehicles basically use the same rule-based control strategy. They differ in calculations of torque distribution due to their different mechanisms. The torque distribution for the proposed MMT vehicle is given in section 3.2, and that for the "THS IIlike" vehicle is calculated based on the fundamental torque and speed formulas of planetary gearsets, which are applied to several EVTs with one or more planetary gearsets [15].

In Fig. 5, vehicle velocity, operation mode, engine power, electric machine (EM, EM1 and EM2) power, battery power and SOC are illustrated. The black solid line represents the results of the proposed MMT vehicle, and the blue or red dashed line of the "THS II-like" vehicle.

It can be seen from Fig. 5(a) that both the MMT vehicle and the "THS II-like" vehic le track the velocity designated by NEDC very well. This indicates that both the vehicles gain identical kinetic energy through the NEDC process.
Fig. 5(b) shows that 10 of the 16 operation modes are used. These operation modes cover 4 of the 5 power flow modes. Compound_1 mode is not applied as the simulation sets the same value for $v_{-}$em_max and $v 1_{1} \max$, below which Motor_only mode is used, and above which Compound_2 mode is used for improving engine efficiency. Other than that, Engine_only_1 is not applied because the required torque does not fall into the engine high or sub-high efficiency region when the vehicle velocity is below $v_{1 \_}$max and the battery SOC is within the lower and upper boundaries during the NEDC simulation.

Since the regenerative braking combined with the mechanical braking can provide the required braking torque, only Reg_braking and Compound_braking_0 mode are applied. It is unnecessary to introduce engine resistance to assist braking, therefore, Compound_braking_1, 2 and 3 mode are not used.

The Charging while parking mode is not activated because the battery SOC can be balanced during vehicle running.

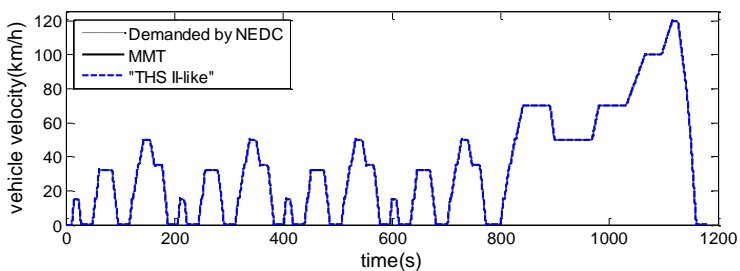

(a)

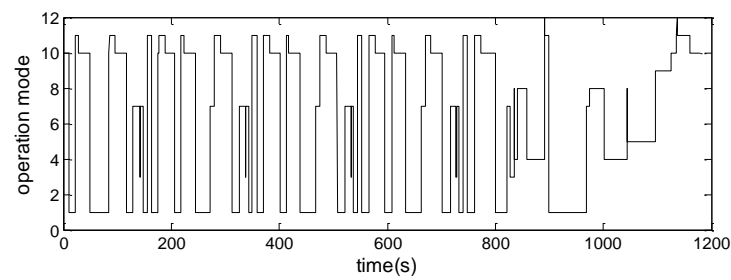

(b)

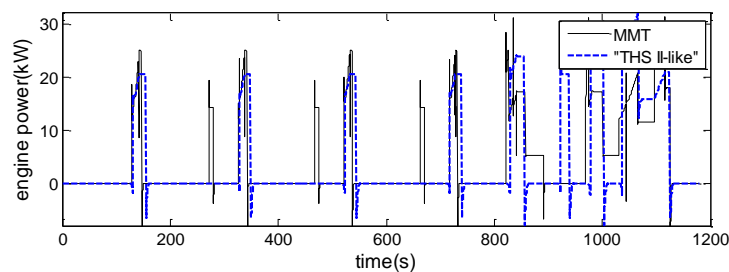

(c)

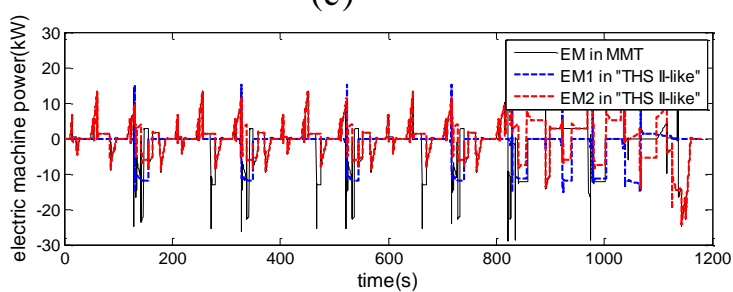

(d) 


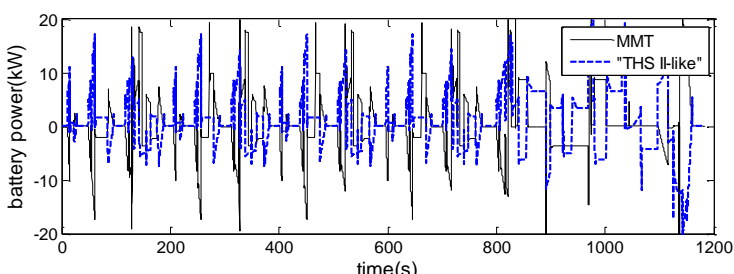

(e)

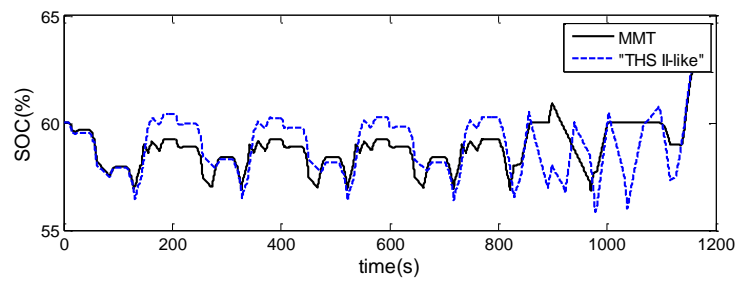

(f)

Figure5: Simulation results of NEDC. (a) Vehicle velocity. (b) MMT operation mode. (c) Engine power. (d) Electric machine power. (e) Battery power. (f) Battery SOC.

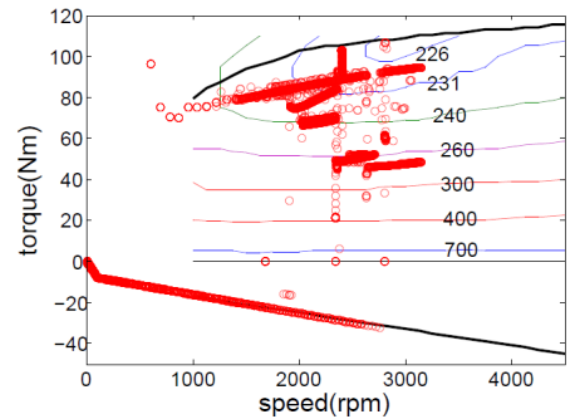

(a)

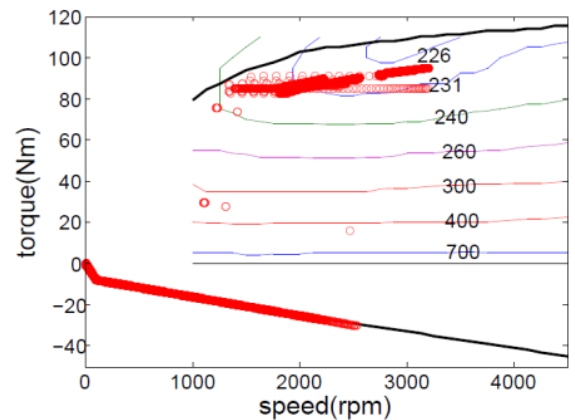

(b)

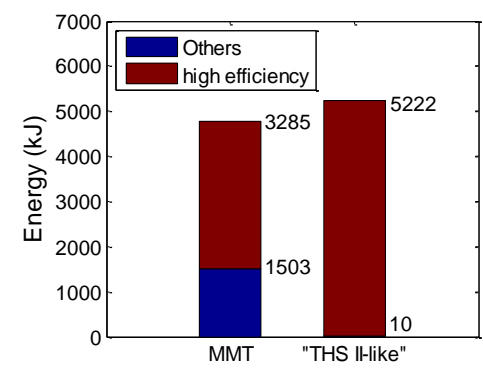

(c)
Figure6: Engine operating points and accumulative energy. (a) MMT vehicle. (b) “THS II-like" vehicle. (c) Fuel-produced energy.

The engine operating points of the MMT vehicle are sketched in Fig. 6(a). Aside from the points with negative torques which occur when the engine is start-up or going to be resting without fuel supply, most operating points spread in the high efficiency region defined by the engine speed from $1500 \mathrm{rpm}$ to $4000 \mathrm{rpm}$ and the engine torque from $70 \mathrm{Nm}$ to $100 \mathrm{Nm}$; at the same time, some points scatter in the sub-high efficiency region with engine torque from $40 \mathrm{Nm}$ to $70 \mathrm{Nm}$. As a comparison, the engine operating points of the "THS II-like" vehicle concentrate on the high efficiency region, as shown in Fig. 6(b). The specific fuel consumptions of these operating points are below $240 \mathrm{~g} /(\mathrm{kWh})$, whereas those of the MMT vehicle are up to $260 \mathrm{~g} /(\mathrm{kWh})$. The vehicle fuel economy is affected by the engine specific fuel consumption and other factors. The next section gives detail explication.

\subsection{Factors Influencing Fuel Consumption}

Over the whole NEDC cycle, the proposed MMT vehicle achieves a fuel consumption of 3.81 $\mathrm{L} / 100 \mathrm{~km}$, and that of the "THS II-like" vehic le is $3.90 \mathrm{~L} / 100 \mathrm{~km}$. The results show that the fuel economy of the two vehicles is comparable, and the MMT vehicle gains minor advantage.

Fig. 6(c) illustrates the accumulative fuel-produced energy from the engine. For the MMT vehicle, the energy produced by fuel combustion all along NEDC is totally accumulated to $4788 \mathrm{~kJ}$, in which, $3285 \mathrm{~kJ}$ comes from the high efficiency operating points, and 1503 from sub-high and other points. However, for the "THS II-like" vehicle, the accumulative energy is totally up to $5322 \mathrm{~kJ}(534 \mathrm{~kJ}$ more than in the MMT vehicle), in which, $5222 \mathrm{~kJ}$ from the high efficiency points, and only $10 \mathrm{~kJ}$ from other points.

It is interesting to reveal a phenomenon that high efficiency usage of the engine does not definitely lead to low fuel consumption of the vehicle. There should be factors other than the engine efficiency affecting the fuel consumption. Possible explanation is, more braking regeneration as supplement energy source or(and) less internal losses. The internal losses come from the electric machines, battery and their power electronics. The following paragraphs give the statistic data and detail explanation for the influence of these factors. 


\subsubsection{Influence of Regenerative Braking}

The accumulative regenerative energy of the MMT vehicle is $916.8 \mathrm{~kJ}$, whereas that of the "THS II-like" vehicle is $815.8 \mathrm{~kJ}$. Different operations of engine braking are found to be a major reason for the regenerative energy gap of $101 \mathrm{~kJ}$.

In the MMT vehicle, engine braking is not applied by opening the clutches, i.e., the braking torque is provided by the combination of regenerative braking and mechanical braking. Moreover, regenerative braking is preferred and mechanical braking is supplement to supply enough braking torque according to the control rules. Thus, engine braking does not consume any regenerative energy from the vehicle body, and the regenerative energy can flow to the battery as much as possible.

However, in the "THS II-like" vehicle, engine braking is inevitable due to the no clutch structure, even if regenerative braking and mechanical braking are applied simultaneously. Negative engine power is recognized in the braking process as shown in Fig. 5(c).

By integral calculation, engine braking energy in the "THS II-like" vehicle is accumulated to $56.64 \mathrm{~kJ}$, whereas that in the MMT vehicle is only $19.02 \mathrm{~kJ}$ (which comes from the kinetic energy of the engine itself). In other words, engine braking in the "THS II-like" vehicle dissipates 37.62kJ more than that in the MMT vehicle. Other reason for the $101 \mathrm{~kJ}$ regenerative energy gap is related to power electronics efficiency, which is discussed in the following paragraphs.

\subsubsection{Influence of the Electric Machines and Their Power Electronics}

It can be seen from Fig. 5(d) that the intervals with zero power of EM in the MMT vehic le are much more than those in the "THS II-like" vehicle. In the MMT vehicle, open or lock operations of the clutches allow efficient usages of EM, whereas in the "THS II-like" vehicle, EM1 and EM2 always rotate when vehicle is running. EM1 must provide a balance torque and EM2 has to rotate simultaneously with the output shaft. As a consequence, the more frequent usage of EM1 and EM2 means more power conversions which lead to more losses. Since an electric machine and its corresponding power electronics, i.e., an inverter, are working simultaneously, they are considered as one unit in this discussion.

To give quantitative statistics, the accumulative output energy for motor and generator working status are given in Fig. 7(d). It is obvious that, when the electric machines are operated as motors, the accumulative output energy of the EM unit is $950 \mathrm{~kJ}$ less than the summation of the EM1 and EM2 unit; when they are operated as generators, the accumulative energy of the EM unit is $1463 \mathrm{~kJ}$ less than the summation. In other words, the EM unit of the MMT vehicle is used less than the EM1 and EM2 unit of the "THS II-like" vehicle.

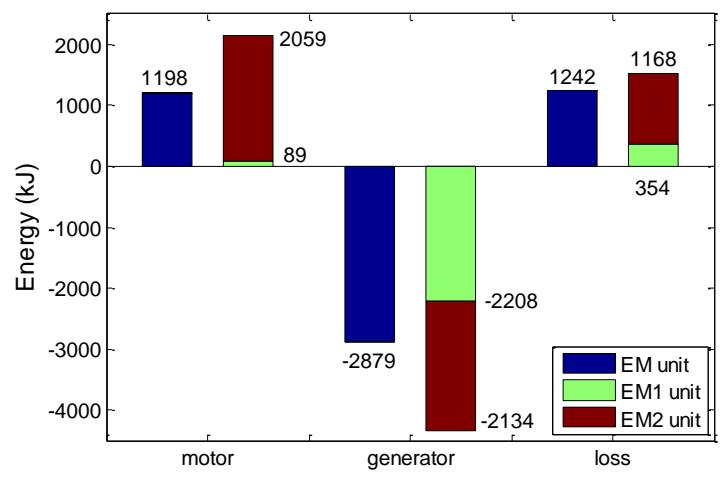

Figure 7: Accumulative energy of electric machine units

The energy loss through the EM unit is $1242 \mathrm{~kJ}$, and that through the EM1 and EM2 unit is $1522 \mathrm{~kJ}$,. Therefore, the EM unit reduces the energy loss by $280 \mathrm{~kJ}$ for the MMT vehicle.

\subsubsection{Influence of the Battery}

Fig. 5(e) shows the battery charging and discharging operations of the "THS II-like" vehicle is more frequent than those of the MMT vehicle. It can be seen from Fig. 8 that 1076kJ less energy is charged into, and $844 \mathrm{~kJ}$ less energy is discharged from the battery of MMT vehicle.

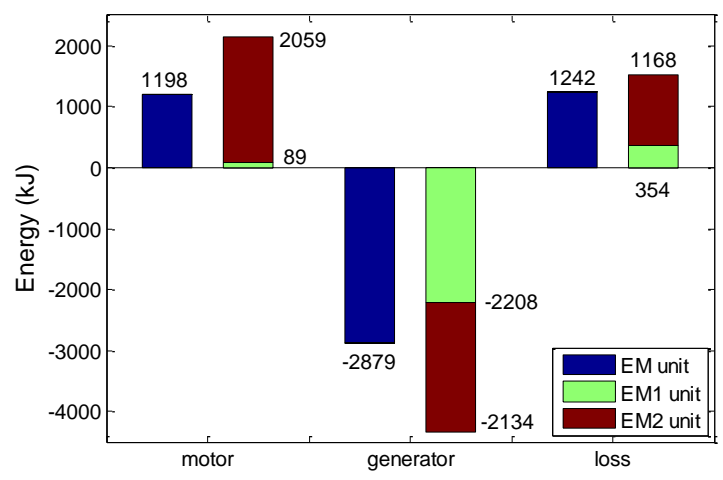

Figure8: Accumulative energy of the batteries

In addition, the battery charging depth of the "THS II-like" vehicle is deeper than that of the MMT vehicle, as shown in Fig. 5(f). Frequent and deep usage of the battery leads to more energy losses (thermal dissipation in Joule) on the battery. Fig. 8 
shows the energy loss through the battery in the MMT vehicle is $268 \mathrm{~kJ}$, and that through the "THS II-like" vehicle is $493 \mathrm{~kJ}$. In other words, the battery of the MMT vehicle reduces the energy loss by $225 \mathrm{~kJ}$.

\subsubsection{Synthesis Analysis}

From the above analysis, three main aspects are found to compensate the $534 \mathrm{~kJ}$ less energy output from the engine of the MMT vehicle: (1) $37.62 \mathrm{~kJ}$ more braking regenerative energy; (2) $280 \mathrm{~kJ}$ less loss in the electric machine units; and (3) $225 \mathrm{~kJ}$ less loss in the battery. Thus, due to the limitation of the single electric machine structure, the MMT vehicle operates the engine in relative lower efficiency region, but achieves less loss from the electric power devices; on the contrary, the "THS II-like" vehicle operates the engine in ideally high efficiency region, but produces more loss from the electric power devices. Therefore, the fuel consumption of the MMT vehicle with proper control strategy can be comparable to that of the "THS II-like" vehicle.

\section{Conclusions}

A MMT combining two planetary gearsets, four clutches and a single EM is proposed in this paper for a HEV. The single EM solution avoids losses from another EM and its power electronics which are employed in many existing HEVs. The MMT proposed here is very similar to the planetary gearsets used in several existing 4speed automatic transmissions, and can have sixteen operation modes which are classified into five power flow modes, Motor_only driving, Engine_only driving, compound driving, braking and charging while parking. A rule-based energy management control strategy, using the efficiency maps of the components for estimating the power performance of the HEV is presented. Simulation under NEDC with same initial and close SOCs shows comparable fuel economy performance to "THS II-like" vehicle, which indicates the fuel economy potential of this concept. Two factors are considered for the efficiency advantage of the single electric machine HEV over the dual electric machine HEV. First, the energy conversion loss in electric components can be reduced due to the existence of Engine_only modes. Second, clutches are used to avoid spin loss of the engine and electric machines.

Notice that due to the no-clutch structure, "THS II-like" vehicle does not allow direct mechanical power transmission from the engine to the drive shaft. Even though the engine can be modulated to its high efficiency region, the overall system efficiency is still lower than the engine only driving under some driving conditions. The fundamental reason is that, the fuel efficiency improvement is counterbalanced by the energy loss in the power conversions among mechanical and electrical components.

\section{Acknowledgments}

The authors would like to thank Chinese National Science Foundation (Grant No. 51105244) and Clean Vehicle Consortium of US-China Clean Energy Research Center (No. 2010DFA72760-305) for the support on this project.

\section{References}

[1] C. C. Chan, The State of the Art of Electric, Hybrid, and Fuel Cell Vehicles, Proceedings of the IEEE, vol. 95, no. 4, pp. 704-718, April 2007.

[2] J. D. Wishart, Y. Zhou, Z. Dong, Review, Modeling and Simulation of Two-mode Hybrid Vehicle Architecture, Proceedings of IDETC/CIE 2007, Las Vegas, Nevada, USA, 2007.

[3] R. H. Staunton et. AL., Evaluation of 2004 Toyota Prius hybrid electric drive system, U.S. Department of Energy Freedom CAR Vehicle Technologies, Washington, DC, ORNLTM-2006/423, May 2006.

[4] A. Takasaki et. AL., Development of New Hybrid Transmission for 2009 Prius, Proceedings of EVS24 International Battery, Hybrid and Fuel Cell Electric Vehicle Symposium, Stavanger, Norway, 2009.

[5] M. R. Schmidt, Two-mode, input-split, parallel, hybrid transmission, U.S. Patent 5,558,588, Sept. 24, 1996.

[6] L. W. Tsai, G. A. Schultz, A MotorIntegrated Parallel Hybrid Transmission, Transactions of the ASME, Journal of Mechanical Design, vol. 126, pp. 889-894, Sept. 2004.

[7] Y. Zhang, H. Lin, Performance modeling and optimization of a novel multi-mode hybrid powertrain, Transactions of the ASME, Joumal of Mechanical Design, vol. 128, pp. 79-89, Jan. 2006.

[8] M. Austin, 2012 Infiniti M35h/M Hybrid PrototypeDrive, http://www.caranddriver.com, accessed on 2011-09-01. 
[9] Simona, 2012 Audi A6 Hybrid, http://www.topspeed.com/cars/audi/2012audi-a6-hybrid-ar101064.html, accessed on 2011-09-01.

[10] T. S. Birch and C. Rockwood, Automatic Transmission \& Transaxles. Englewood Cliffs, NJ: Prentice-Hall, 2009.

[11] Futang Zhu, Li Chen, Chengliang Yin, Design and analysis of a novel multi-mode transmission for a HEV using a single electric machine, IEEE Transactions on Vehicular Technology 2013, vol. 62, no. 3, pp. 1097-1110.

[12] W. Xiong, Y. Zhang, C. Yin, Optimal energy management for a series-parallel hybrid electric bus, Energy Conversion and Management, vol. 50, no. 7, pp. 1730-1738, March 2009.

[13] J. Liu, H. Peng, Modeling and control of a power-split hybrid vehicle, IEEE Transactions on Control Systems Technology, vol. 16, no. 6, pp. 1242-1251, Nov. 2008.

[14] J. Moreno, M. E. Ortúzar, and J. W. Dixon, Energy-Management System for a Hybrid Electric Vehicle, Using Ultracapacitors and Neural Networks, IEEE Transactions on Industrial Electronics, vol. 53, no. 2, pp. 614-623, April 2006.

[15] L. Chen, F. Zhu, M. Zhang, Y. Huo, C. Yin, and H. Peng, Design and analys is of an electrical variable transmission for a seriesparallel hybrid electric vehicle, IEEE Transactions on Vehicular Technology, vol. 60, no. 5, pp. 2354-2363, June 2011.

\section{Authors}

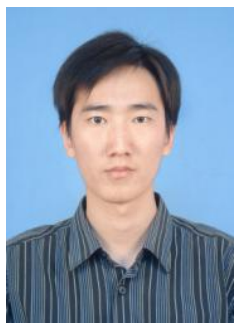

Futang Zhu was bom in Shandong, China, in 1984. He received the B.S. degree in vehicle engineering from Jilin University, Changchun, China, in 2007. He is currently working toward the Ph.D. degree with the School of Mechanical Engineering, Shanghai Jiao Tong University, Shanghai, China.

From 2011 to 2012, he was a visiting scholar at ETAS GmbH, BOSCH Group in Stuttgart, Germany. His research interests include modelling and control of electric vehicles, particularly for power coupling mechanisms of hybrid electric vehicles, as well as the model-based ECU development using V-cycle.

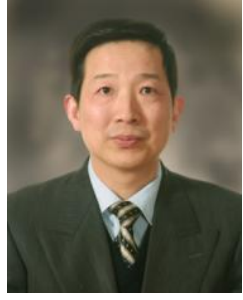

Chengliang Yin was bom in Inner Mongolia, China, in 1965. He received the Master and Ph.D. degree in vehicle engineering from Jilin Industrial University, China, in 1996 and 2000, respectively.

$\mathrm{He}$ is currently a Full Professor of Shanghai Jiao Tong University, where he is also the Vice Dean of Institute of Automotive Engineering as well as the Vice Director of National Engineering Laboratory for Automotive Electronic Control Technology. His research interests include control of automotive electronics, electric vehicles, especially the research and development of hybrid electric vehicles.

Prof. Yin is employed as the advanced technical adviser of the Shanghai Automobile and Dongfeng Automotive group. In 2009, Prof. Yin was honored as the General Motors Innovative Talent in Automotive Industry.

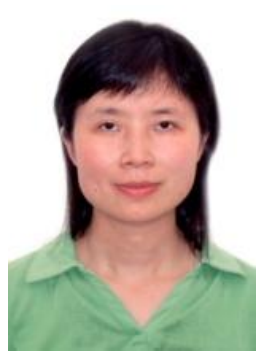

Li Chen was born in Hunan, China, in 1973. She received the B.S. and M.S. degrees in mechanical engineering from Hunan University, Changsha, China, in 1994 and 1997, respectively; Ph.D. degree in vehicle engineering from Shanghai Jiao Tong University, Shanghai, China, in 2000.

She is currently an Associate Professor with the School of Mechanical Engineering, Shanghai Jiao Tong University, Shanghai, China. Her research interests include dynamics, control and thermal performance for advanced powertrain systems. 